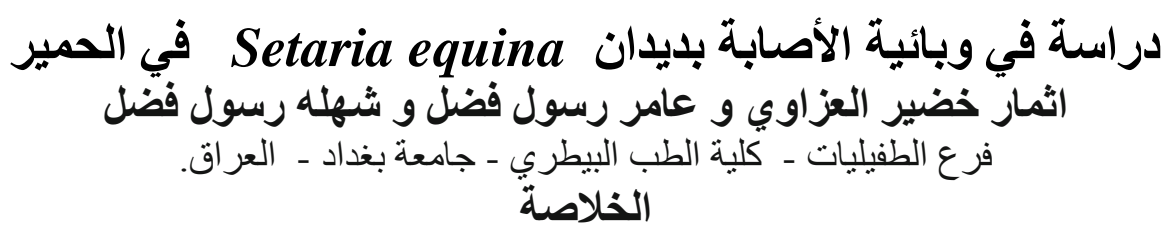

تم فحص 218 حيو ان (106 ذكور و 112 اناث) من الحمير المذبوحة في حديقة حيو انات الزور إهاء من مناطق وسط

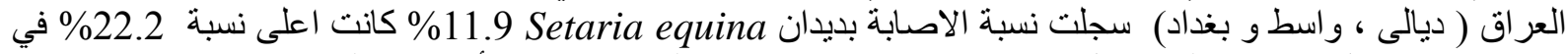

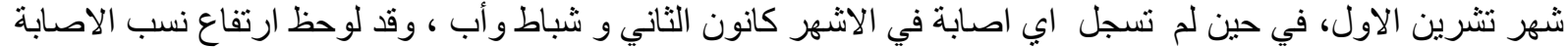

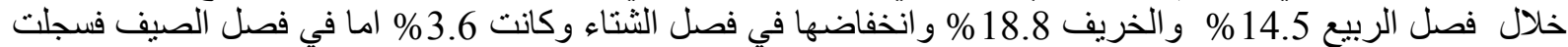

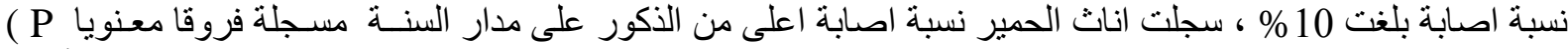

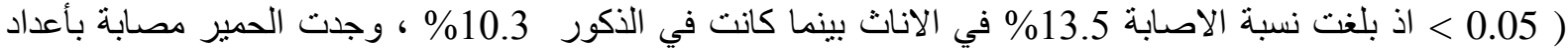

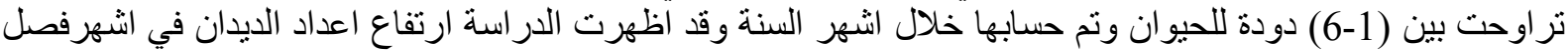

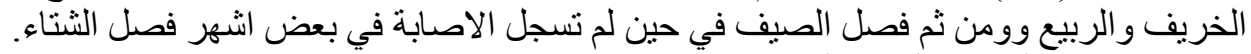

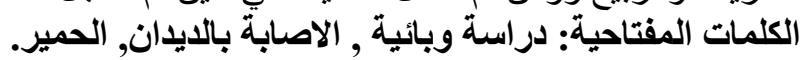

\title{
Epidemiological study of Setaria equina infection in donkeys
} Athmar .K. AL-Azawi, Amer Rasool Fadhl and Shahela Rasool Fadhl Department of parasitology, College of Veterinary Medicine, Baghdad University, Iraq. Accepted: 14/2/2012

Summary

Autopsy study of 218 (106 male and 112 female) animals slaughtered in Baghdad ( AlZAWRA ) Zoo, of central Iraq (Diyala, Wasit, Baghdad). The total infection rate of Setaria equina was $11.9 \%$ and the highest infection rate $(22.2 \%)$ was recorded in October, where is no infection in January, February and August. It has been observed that the high infection rate $(14.5 \%)$ during the months of spring and autumn (18.8\%) and decrease in winter and summer $3.6 \%$ and $10 \%$. Female donkeys with a higher proportion $(13.5 \%)$ than male $10.3 \%$ respectively differences ( $\mathrm{P}<0.05$ ). Worm intensity ranged between 1-6 worm and was shown high worm burden in Autumn and Spring fallowed by Summer and finally Winter seasons, which no infection was recorded in some months of it

Keywords: Setaria equine, donkeys, Epidemiology, infection.

\section{المقدمة}

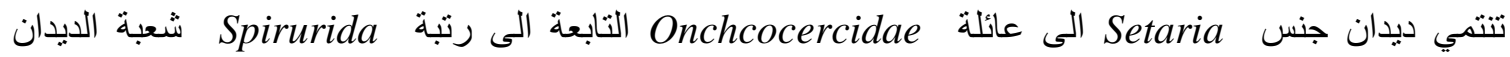

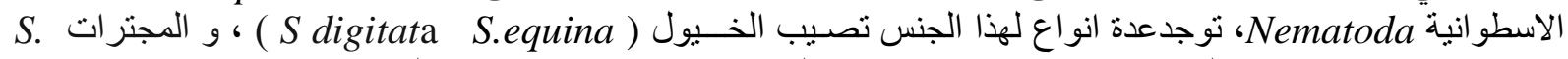
marshalli

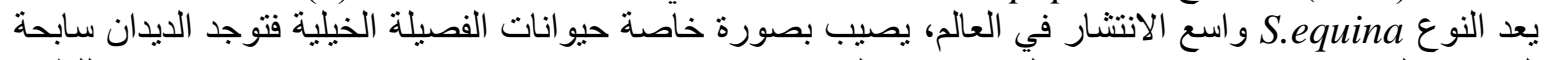
في التجويف البريتون (4 و5) .ان انواع البعوض من الجنسين (Culex Anopheles و (6)، تعد ناقاقلات جيدة للطور

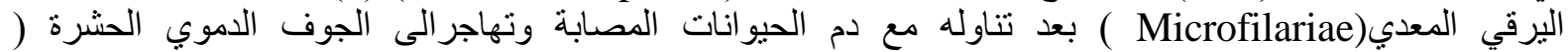
(haemocoel الدهنية و عضلات الطيران (Flight muscles) ونبيبات ماليبجي اذ تعاني الانسلاخ ويحصل تنطور الى اليرقة الئنة الثانية

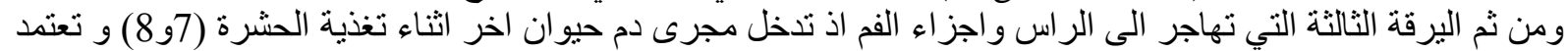

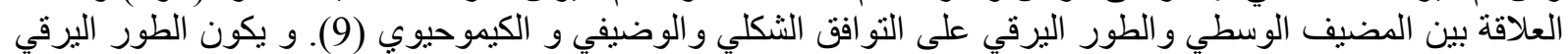
نسبيا غير مؤذي للوسيط الناقل مالم يتو اجد بأعداد كثيرة.

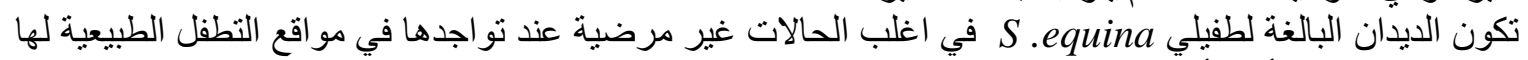

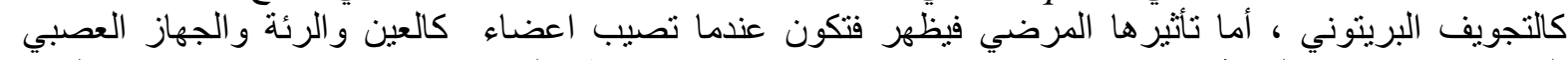

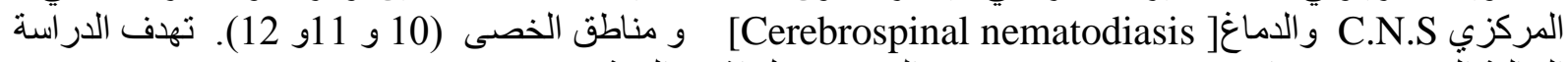
الحالية التحري عن ديدان Setaria equina في الحمبر خلال اشهر السنة. . 


\section{المواد وطرائق العمل}

تم فحص 218 حيوان (106 ذكور و112 اناث ) وبمعدل 5 حيو انات اسبو عيا من الحمير المذبوحة في حديقة حيو انات الزور اء و التي تجلب اليها من مناطق وسط العراق ( ديالى وواسط وبغداد) للفترة من كانون الإن الاول

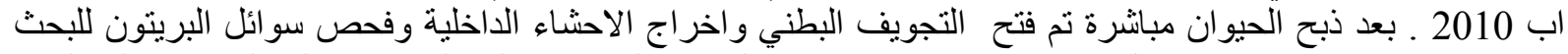

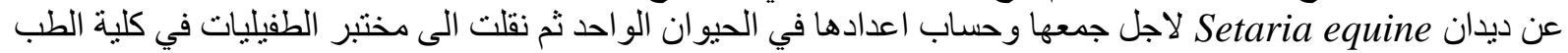

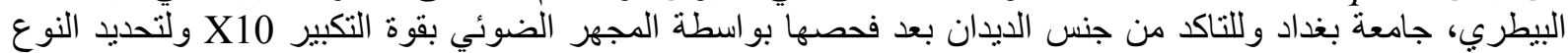

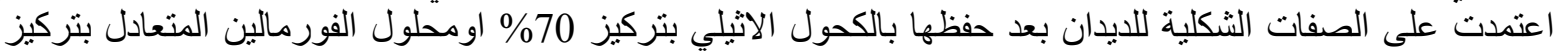
10 buffered formalin تم تحليل البيانات بواسطة الحاسبة الالكترونية باستخدام البرنامج الاحصائي SAS. (14).

\section{النتائسج و المناقشتة}

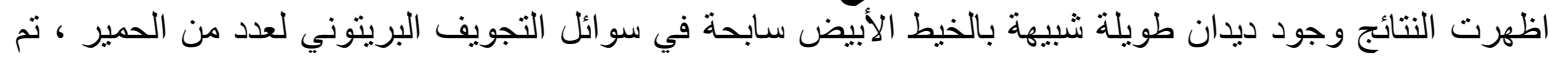

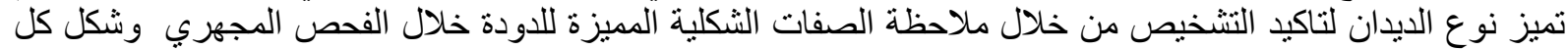

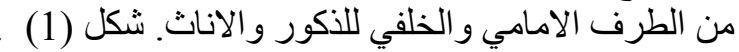

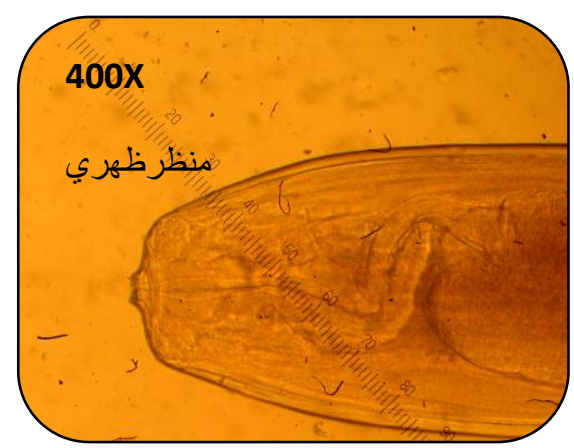

(ب).الطرف لامامي للطفيلي.

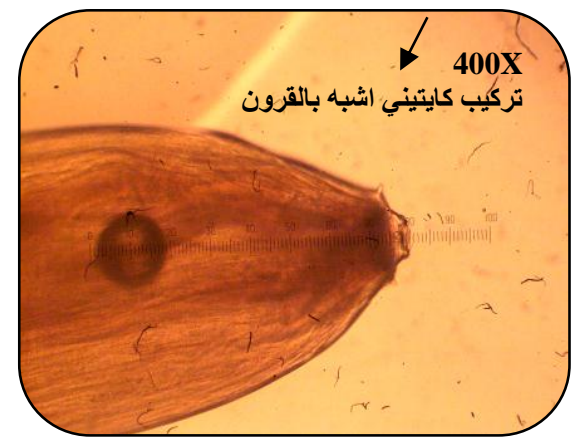

(د)(الطرف الامامي للطفيلي. . (cuticular ring).

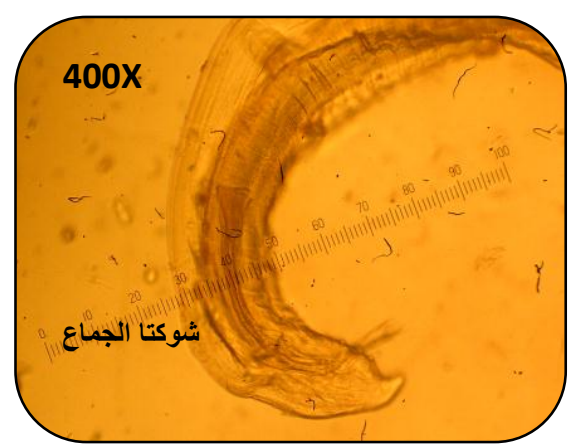

(أ) .الطرف الخلفي لذكر الطفيلي

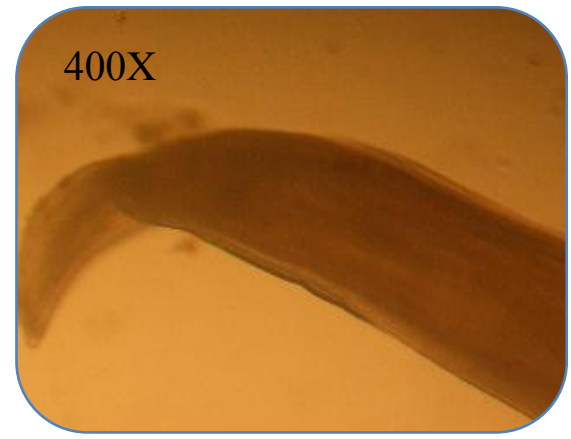

(ج) الطرف الخلفي لأنتى الطفيلي.

\section{شكل (1) ـ الطرف الأمامي والخلفي للطفيلي Setaria equina مامبل}

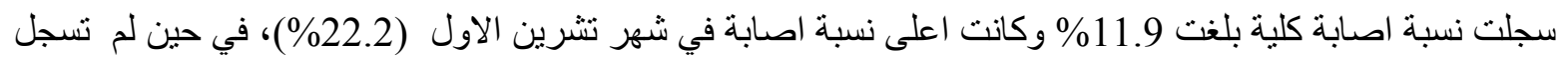

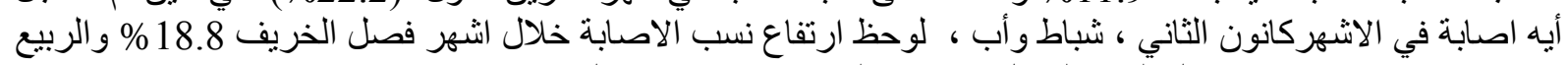

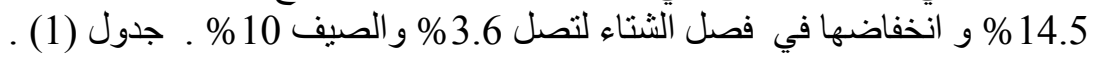




\section{جدول (1) ـ اعداد الحيوانات ونسب الاصابة بديدان Setaria equina في الحميرخلال اشهر وفصول السنة .}

\begin{tabular}{|c|c|c|c|c|c|c|c|}
\hline & & & & \multicolumn{2}{|c|}{ الاكلي } & \multicolumn{2}{|c|}{ فصول واشهر السنة: } \\
\hline & $\%$ & & مصد & & مفحو & & \\
\hline$\% 11$ & 3.6 & 2 & 2 & 18 & 55 & 15 & \\
\hline - & & & 0 & 17 & & ك5 & \\
\hline- & & & 0 & 20 & & شباط & \\
\hline 11.7 & 14.5 & 8 & 2 & 17 & 55 & أذار & \\
\hline 11 & & & 2 & 18 & & نيسان & ; \\
\hline 20 & & & 4 & 20 & & أيار & \\
\hline 17.6 & 10 & 6 & 3 & 17 & 55 & حزيران & \\
\hline 16.6 & & & 3 & 18 & & تموز & $0^{\circ}$ \\
\hline- & & & 0 & 20 & & اب اب & \\
\hline 16.6 & 18.8 & 10 & 3 & 18 & 53 & ايلول & \\
\hline 22.2 & & & 4 & 18 & & $1 ت$ & $7^{2}$ \\
\hline 17.6 & & & 3 & 17 & & ت & \\
\hline & 11.9 & & 26 & & 218 & المجموع & \\
\hline
\end{tabular}

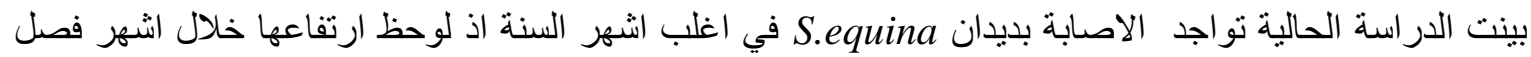

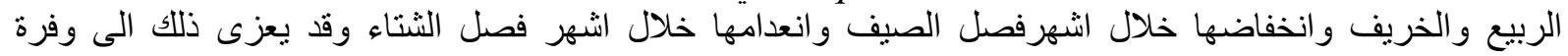

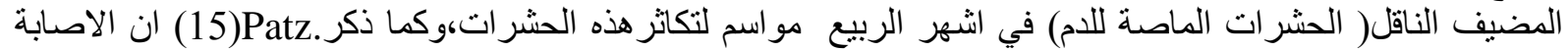

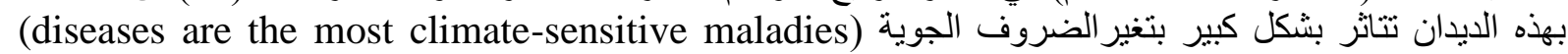

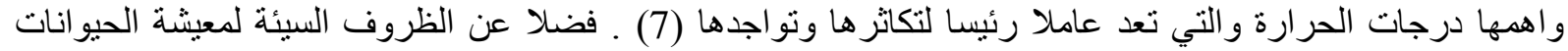

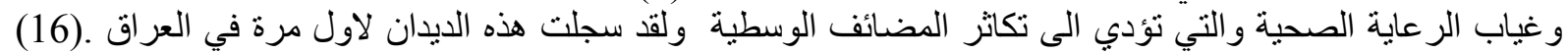

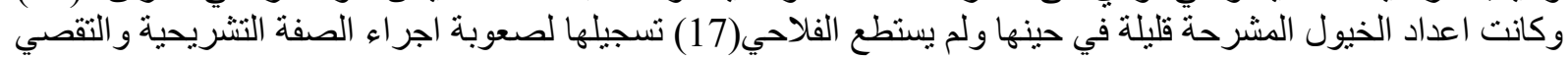

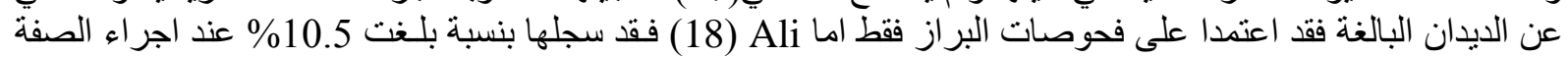

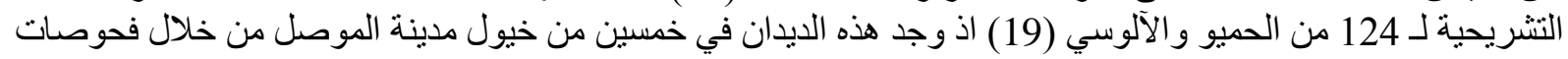

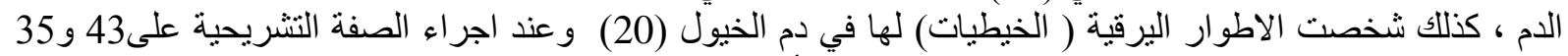

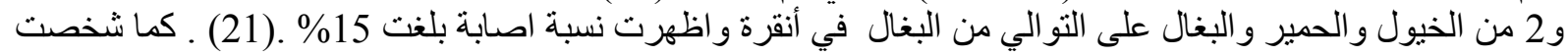

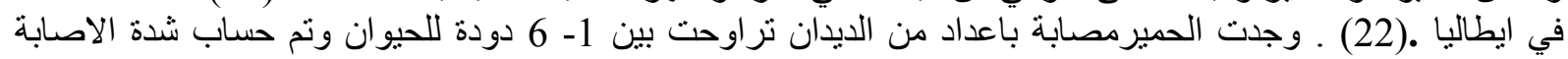

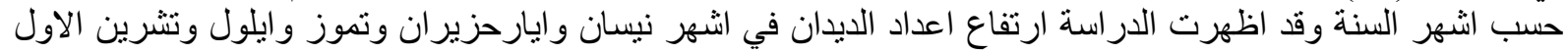
و انخفاضها في اشهر اذار وتشرين الثاني وكانون الاول وانعدامها في اشهر كانون الثناني وشباط واب واب ـ ـ جدول (2).

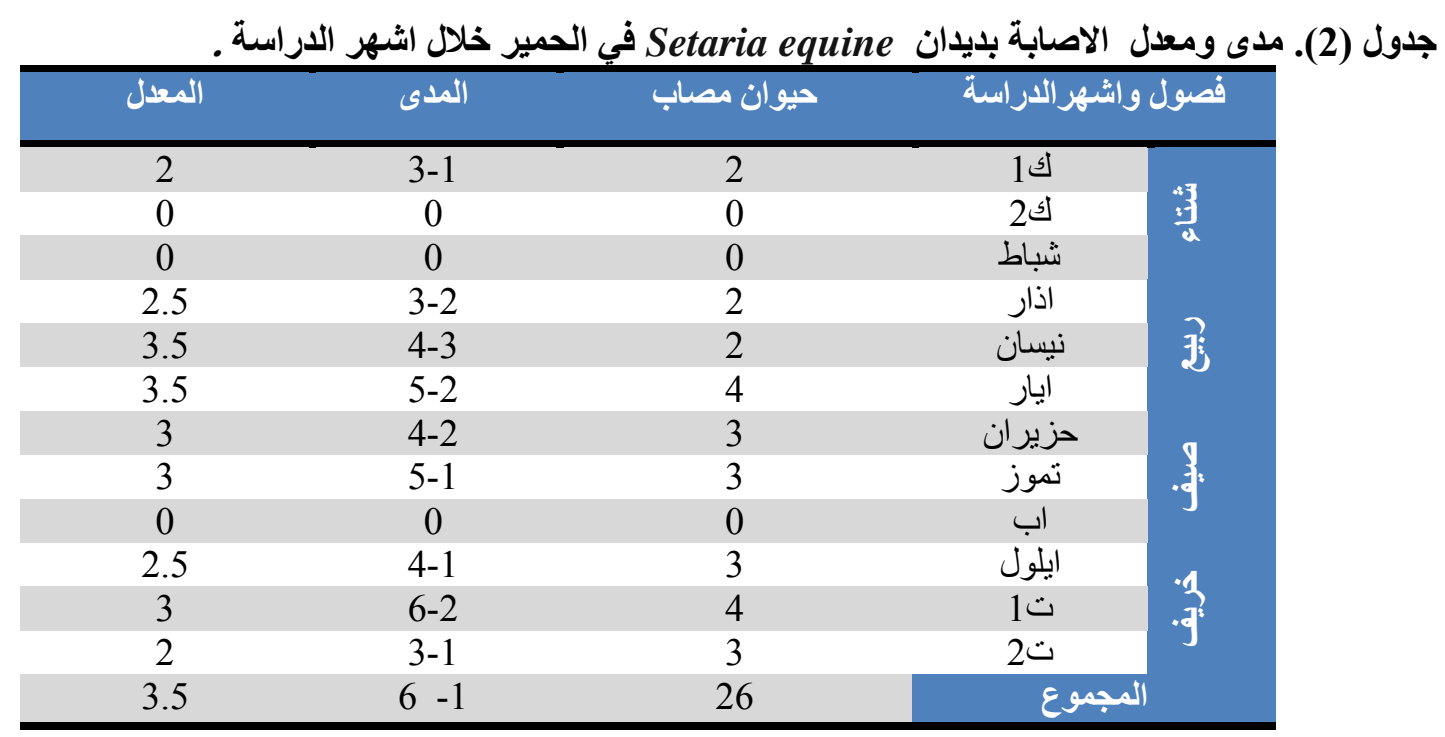


سجل دراسة Ali (18) شدة اصابة تراوحت ( 19-19) دودة وكانت اعلى مقارنة بنتائج الدراسة الحالية وقد اشار التار

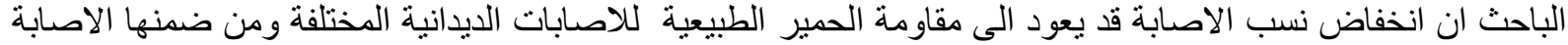
بديدان S.equina فضلا عن تقارب نسب الاصابة مقارنة قد يعزى الحيو انات الى ان معظم الحيو انات كانت ضمن الفئة العمرية 2-4 سنة .

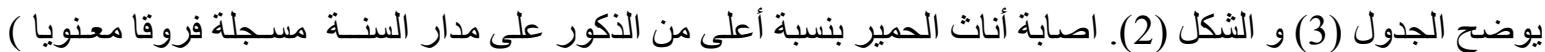

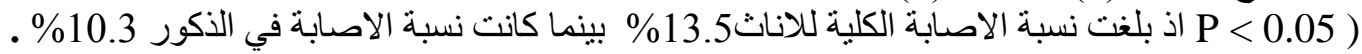

$$
\text { جدول ( } 3 \text { ).اعداد ونسب اصابة ذكور واناث الحمير خلال اشهر الاراسة . }
$$

\begin{tabular}{|c|c|c|c|c|c|c|c|c|}
\hline المئوية & ذكور & كأكمر & المنيبة & مصاب & انثاث & كلياب & الكالي & الاشثهر \\
\hline 0 & 0 & 8 & $20 \%$ & 2 & 10 & 2 & 18 & 1 \\
\hline 0 & 0 & 8 & 0 & & 9 & 0 & 17 & 15 \\
\hline 0 & 0 & 10 & 0 & 0 & 10 & 0 & 20 & شباط \\
\hline $11.1 \%$ & 1 & 9 & $12.5 \%$ & 1 & 8 & 2 & 17 & اذذار \\
\hline $11.1 \%$ & 1 & 9 & $11.1 \%$ & 1 & 9 & 2 & 18 & نيسان \\
\hline $20 \%$ & 2 & 10 & $20 \%$ & 2 & 10 & 4 & 20 & ايار \\
\hline $11.1 \%$ & 1 & 9 & $25 \%$ & 2 & 8 & 3 & 17 & حزيران \\
\hline $25 \%$ & 2 & 8 & $10 \%$ & 1 & 10 & 3 & 18 & تموز \\
\hline 0 & 0 & 10 & 0 & 0 & 10 & 0 & 20 & اب \\
\hline $11.1 \%$ & 1 & 9 & $22.2 \%$ & 2 & 9 & 3 & 18 & ابلول \\
\hline $22.2 \%$ & 2 & 9 & $22.2 \%$ & 2 & 9 & 4 & 18 & ت1 \\
\hline $12.5 \%$ & 1 & 8 & $22.2 \%$ & 2 & 9 & 3 & 17 & 2 \\
\hline $10,2 \%$ & 11 & 107 & $13.5 \%$ & 15 & 111 & 26 & 218 & المجموع \\
\hline
\end{tabular}

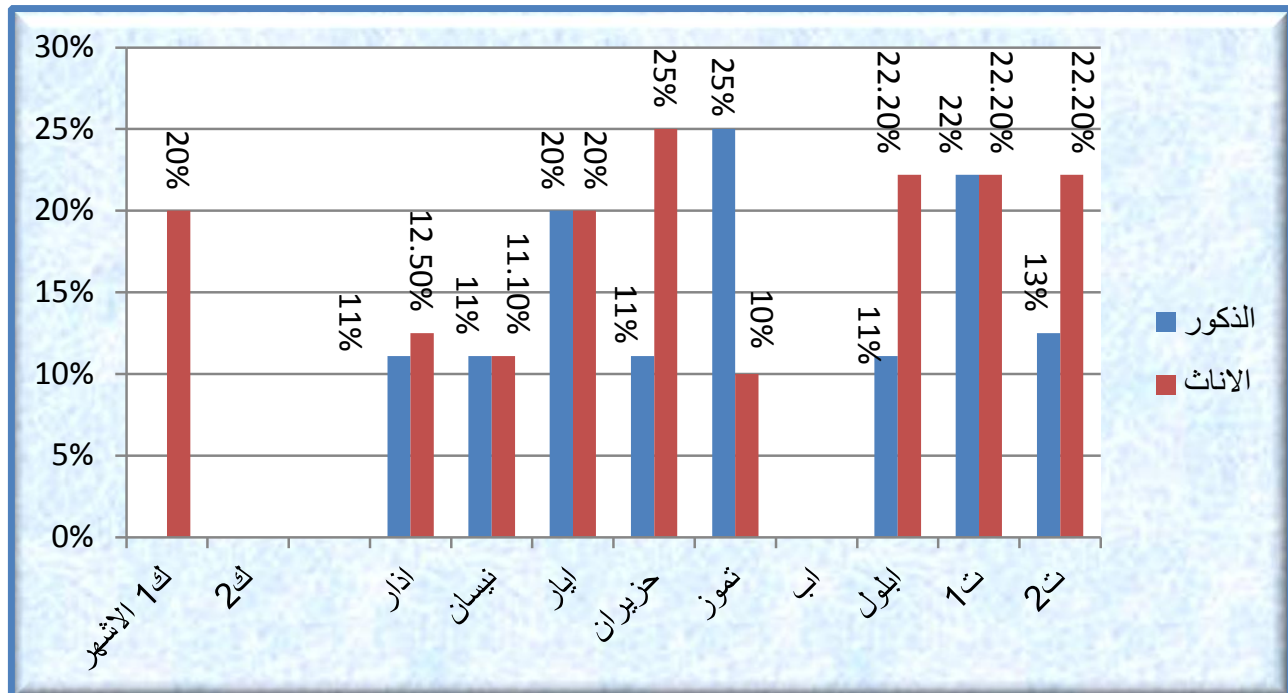

شكل.(2). نسب اصابة ذكور و اناث الحميرخلال اشهر السنة .

سجل Ali) في بغداد في دراسته تفوق نسب الاصابة في جنس الاناث عنه في الذكور لتاثثر عوامل الاجهاد من

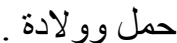
ان ديدان جنس Seteria تمثل اهمية اقتصادية خصوصا عندما تتطفل على الحيو انات الحقلية لما تسببه من امر اض مثل

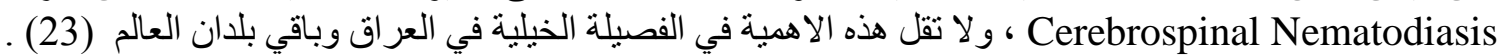

\section{المصادر}

1. Yadav, A.; Kumar, A.; Bhadwal, MS.; Khajuria, JK., and Gupta, A. (2006). Ocular setariosis in horses: A case study. Vet. Parasitol., 20: 2-5. 
2. Jemelka, ED.( 1976). Removal of Setaria digitata from the anterior chamber of the equine eye. Vet. Med. Small Anim. Clini., 71: 673-675.

3. Panaitescu, D., Preda, A., Bain, O. and Bugarin, A., (1999). Four cases of human filariasis due to Setaria labiatopapillosa in Bucharest, Romania. Roumania Archie. Micro. and Immuno., 58: 203-207.

4. Coleman, SU.; Klei, TR. and Fremch, DD.,(1985). Prevalence of Setaria equine (Nematode : Onchocercidae)in Southeastern Louisiana horses .J. Parasito., 71:512-513.

5. Radostits, OM.; Gay, CC.; Blood, DC. and Hinchcliff, KW. (2000). A textbook of the diseases of cattle, sheep, pigs, goats and horses. $9^{\text {th }}$ Ed. WB Saunders Co, pp. 1339-1386.

6. World Health Organization (2007) WER. 82:361-380.

7. Anderson, RC. (2000).The Superfamily Filarioidea. In Nematode Parasites of Vertebrates; Their Development and Transmission. $2^{\text {nd }}$ Ed. New York: CABI Publis., PP:467-529.

8. Rehbinder, C. (1990). Some vector borne parasites in Swedish reindeer (Rangifer tarandus). Acta. Vet. Scand, 10:67-73.

9. Bain, O. and Babayan, S.(2003). Behavior of filariae: Morphological and anatomical signatures of their life style within the arthropod and vertebrate hosts. Filarial J., 2:16-20.

10. Bazargani, T.; Eslami, A. and Ghoumi, G.( 2008).Cerebrospinal Nematodiasis of cattle ,sheep and in Iran .Iran. Parasitol., 3(1):16-29.

11. Muhammad, G. and Saqib, M. (2007). Successful treatment of ocular equine microfilaria is (setaria sp.) with ivermectin. Vet. Record, 160:25-26.

12. Solusby, EJ. (1982). Helminths, arthropods and protozoa of domesticated animals. $7^{\text {th }}$ Ed. Philadelphia, Bailliere Tindall, London.

13. Burgu, A.; Oge, S.; Doganay, A.; Piskin. C. and Oge, H. (1995). Helminth species found in donkeys. Ankara Univ Vet. Fak. Derg ., 42:193-205.

14. SAS. (2001).SAS/STAT user guide For personal computer .release 6.12.SAS Inst . Inc. Cary .N.C. USA.

15. Patz, JA.; Graczykb, TK.; Gellera, N. and Vittorc, AY. (2000). Effects of environmental change on emerging parasitic diseases. Int. J. Parasitol., 30(1): 395-405.

16. Leiper, JW.(1957). Animal parasites and their control, Report to the Government of Iraq. Rome : FAO,610.

الفلاحي،اياد محمود(1992) در اسة في وبائية الديدان الاسطوانية في المعدة و الامعاء في الخيول في بغداد ،در اسة

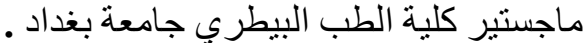

18. Ali, SR.; Rahif, RH. and AL-Kubaisee, RY.(1992). Prevalence of internal parasites amang equine in central Iraq. TheVeterinarian, 2(1):35-41.

الآلوسي، توفيق إيراهيم ، أرسلان، سامح هدايت و زنكنة، إحسان قادر (1994). دراسة بعض الإنة الأخماج الطفيلية في

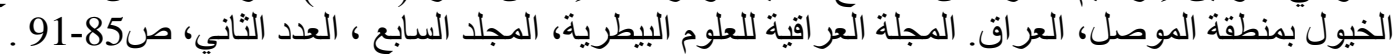

20. Yousif, YA., Haytee, ZG., Saleem, AN. and Johi, HC.(1990) . Haemato- biochemical changes in microfilaria effected horses under field condition. J. Vet. Parasite., 3:2-5 .

21. Oge, S.;Oge, H.; Yildirim, A. and Kircali, F. (2003). Setaria equine infection of Turkish equines estimates of prevalence based on necropsy and the detection of microfilaraemia . Parasitol., 97(4):403-409.

22. Ricci, M., and Sabatini, A. (1992). Parasitic helminthes of the cecum and colon of equidae in Italy. Parassitologia., 34(1-3): 53-60

23. Buchwalder, R., and Schuster, R. (1989). Finding of Setaria equina in horses. Ang. Parasitol., 30(2):127-130. 